\title{
A four-element UWB MIMO antenna using SRRs for application in satellite communications
}

\author{
Chafai Abdelhamid ${ }^{1}$, Hedi Sakli ${ }^{2}$, Nizar Sakli ${ }^{3}$ \\ ${ }_{1,2}^{1,2}$ ACS Research Laboratory, National Engineering School of Gabes, Gabes University, Gabes, Tunisia \\ ${ }^{3}$ EITA Consulting, Montesson, France
}

\begin{tabular}{l} 
Article Info \\
\hline Article history: \\
Received Jan 20, 2020 \\
Revised Jan 4, 2021 \\
Accepted Jan 19, 2021 \\
\hline
\end{tabular}

Keywords:

Diversity gain

ECC

Isolation

Metamaterial

UWB MIMO antenna

$\mathrm{X}$ and $\mathrm{Ku}$ bands

\begin{abstract}
This paper proposes a method for designing a new ultra wide band (UWB) multiple-input multiple-output (MIMO) antenna with two and four elements. First we presented an ultra-wide band antenna we studied these performances. Then, we studied the application of metamaterials to the design of MIMO antennas for miniaturization and the performance of antennas, in order to guarantee the proper functioning of the MIMO system with a much reduced separation distance between the radiating elements $(\lambda / 12)$, where the coupling can be very weak. The application of these circular double ring SRRs materials on the front plan of the antenna has contributed to the increasing of the antenna performance is studied in terms of S-Parameters, efficiency, diversity gain (DG), radiation properties and envelop correlation coefficient (ECC). It offers advantages such as the reduction of weight and congestion that is beneficial for their integration into satellite communications systems.
\end{abstract}

This is an open access article under the CC BY-SA license.

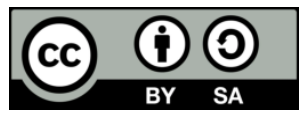

\section{Corresponding Author:}

Hedi Sakli

EITA Consulting

5 Rue du Chant des oiseaux, 78360 Montesson, France

Email: hedi.s@eitaconsulting.fr

\section{INTRODUCTION}

Multi-antenna systems are developing very rapidly, especially in the field of wireless communications. With the emergence of new standards, the wireless industry market [1] is increasingly using multi-antenna multi-access systems in which the antennas work either in the same bands or in different frequency bands. These systems for MIMO and diversity applications help to avoid fading due to the multitrip environment and improve channel capacity [2]. In all communication systems, the antenna is a critical element, whose implementation influences their overall performance. When designing antennas, several parameters must be taken into account (radiation efficiency...) [3-5] that have a huge influence on signal characteristics and transmission or reception quality. The prodigious development of such a printed structure is linked to the considerable progress made in the 1980s in the field of miniaturization, in the integration of electronic circuits and especially low-loss dielectric substrates [6,7].

Numerous applications of microbands antennas such as, mobile telephony, military applications telelocation, the different standards of radio, mobile communications (WIFI, WIMAX, Bluetooth, UMTS, WLAN, 4G, and 5G) as well as space communications, require a very wide UWB bandwidth [8], essentially enabling the compatibility of the different standards on the one hand and access to many services from the same device on the other [9]. Mainly the design of an antenna is done in coincidence, according to the frequency band, the gain, the cost and the weight. Antenna miniaturization techniques are the subject of a lot of short research and development work in recent years and today. A wide variety of UWB monopoly printed 
antennas have been proposed in the literature. Different shapes are presented to ensure ultra-wide band passing with high efficiency, such as rectangular, trapezoid, circular, polygonal, and elliptical antenna. For example, in [10], the author is studying a new antenna, in relative bandwidth reaches $153.22 \%$ and an efficiency of $86 \%$, will be used for Wimax/WLAN and several wireless communication applications. In [11], an antenna was proposed for UWB applications designed for wireless communication applications, with a relative bandwidth (BPR) of approximately $119 \%$ and an efficiency of around $82 \%$. Mutual coupling is an electromagnetic phenomenon likely to occur in antenna arrays. It is due to the electromagnetic interactions that occur between the elements of an antenna array and induce a modification of the intrinsic parameters of the antennas. Therefore, the design of a system containing several antennas in a small volume such as a mobile terminal is more difficult than a single antenna system [9]. The proximity of the antennas can lead to a mutual coupling phenomenon degrading total efficiency, especially in the case of multi standard antennas. In MIMO and/or diversity systems, this coupling will also lead to an increase in the correlation envelope and thus a degradation of the diversity gain and the capacity of the channel. Reducing this mutual coupling is the objective of the theoretical and practical work already done on multi-antenna systems in telecommunications. Several techniques have been adopted to reduce mutual coupling between MIMO elements such as parasitic elements [12,13], electronic band gap (EBG) [11, 12], artificial metamaterials [14, 15], defective ground structure (DGS) [16] and filters are introduced to improve isolation. Recently, the appearance of metamaterials, which is an artificial composite structure that can be presented in several types; the best known are those likely to have both negative permeability and permittivity to be of great importance to researchers in the telecommunications world [16-18]. Metamaterials are used for the design of several devices, (antenna, and filter) [19-21] has caused their advantages as easy integration as well as low manufacturing cost. The solution is to use a structure based on metal rings split ring resonators (SRR) with negative permeability. Cascading SRRs are located along the distance between two adjacent, opposite and even symmetrical UWB antennas based on the FR4 substrate layer to separate the antennas from each other to ensure proper isolation.

\section{UWB ANTENNA DESIGNING}

In the first step, we start our design with a square shape. The choice of this kind of simple element printed on the substrate aims to reduce the size of the structure but also to have an antenna that is easy to produce and inexpensive. For a resonant frequency of $11.2 \mathrm{GHz}$, the dimensions of the rectangular patch can be obtained from the analytical expressions given in [21] by:

$$
L=\frac{c}{2 f \sqrt{\varepsilon_{e f f}}}-2 \Delta L
$$

With

$$
\begin{aligned}
& \Delta L=0.412 h \frac{\left(\varepsilon_{\text {eff }}+0.3\right)\left(\frac{w}{h}+0.262\right)}{\left(\varepsilon_{\text {eff }}-0.258\right)\left(\frac{w}{h}+0.813\right)} \\
& \varepsilon_{\text {eff }}=\frac{\varepsilon_{r+1}}{2}+\frac{\varepsilon_{r}-1}{2}\left(1+\frac{10 h}{w}\right)^{\frac{-1}{2}}
\end{aligned}
$$

The initial width of the antenna is given by the expression (4) [22].

$$
w=\frac{\lambda_{0}}{2}\left(\frac{\varepsilon_{r}}{2}\right)^{\frac{-1}{2}}
$$

We feed the antenna by a coaxial probe, at a point located on its axis of symmetry, more or less close to the edge for the impedance adaptation. The coaxial probe cable passes through the ground plane and the substrate. The central conductor is connected to the radiating element, and the external conductor is connected to the ground plane as shown in Figure 1. The ground is total. The simulation of this structure gave a poor adaptation of the bandwidth in the operating frequency band ( $\mathrm{X}$ and $\mathrm{Ku}$ bands). The antenna design is based on the transformation of a simple rectangular antenna with reduced bandwidth into a structure meeting the bandwidth criterion, by introducing some disturbances on its geometry. It is possible to broaden the bandwidth of an antenna by adding an impedance matching network to its input [23]. It is used to optimize 
the transfer of power between the resonator and the source. Thus, we obtain, naturally, an enlargement in the bandwidth. The techniques used are the insertion of slots or notches which make it possible to modify the paths of the surface currents and therefore of the distribution of impedance along the antenna. This method is interesting since it makes it possible to conserve or even reduce the size of the original antenna [24, 25]. As the total ground plan does not favor the widening of the bandwidth and in order to optimize the design, we will, first of all, carry out a parametric study on the insertion of the rectangular slots. Figure 1 shows the shape and dimensions of the initial antenna composed of a copper rectangular of thickness $35 \mu \mathrm{m}$, of length $\mathrm{Wp}$ and a width of the LP. Figure 2 shows the geometry and dimensions of the modified antenna. Figure 3 presents and mentions the geometry and the dimensions of the final antenna. The substrate used is an FR4epoxy dielectric with relative permittivity $\varepsilon r=4.4$, thickness $h=1.6 \mathrm{~mm}$ and loss tangent $\operatorname{tg}(\delta)=0.02$. The ground plane covers the entire rear face of the substrate. Figure 4(a), illustrates the reflection coefficient of the initial antenna shows that it has a narrow bandwidth ranging from 11 to $11.3 \mathrm{GHz}$ before modification. Figure 4(b), shows the reflection coefficient of the modified antenna shown in Figure 2 as a function of frequency. We notice an improvement in terms of adaptation after the addition of the rectangular slots, with a minimum value of around $-26 \mathrm{~dB}$. To further improve the adaptation of the antenna, cutting on the staircase was involved as well as the use of the slots in the radiating element. These modifications in Figure 3 have not only allowed an extension of the current paths (by forcing them to bypass this slot) but also the creation of new resonances and the enlargement of the bandwidth. The Figure 4(c), illustrates the reflection coefficient of the final antenna as a function of frequency. A considerable decrease in the level of the S11 while expanding the bandwidth is well noticed. The parameters associated with the proposed antenna are provided in Table 1.

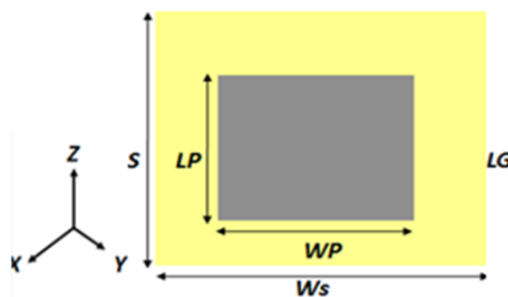

(a)

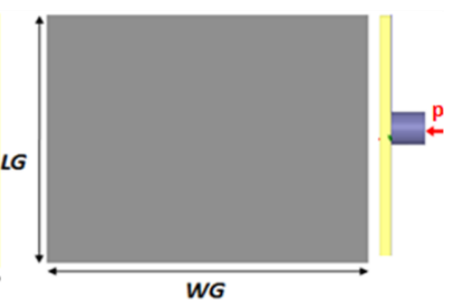

(b) (c)

Figure 1. Initial antenna: (a) In front view (b) bottom view<smiles>[Y]C([Y])[CH]</smiles>

Figure 2. Modified antenna: (a) In front view (b) bottom view

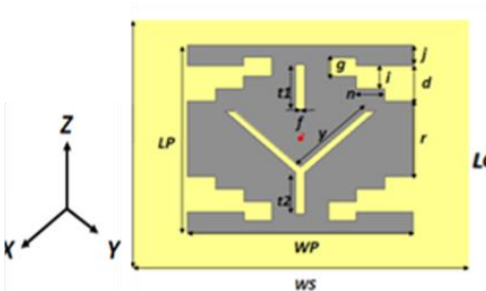

(a)

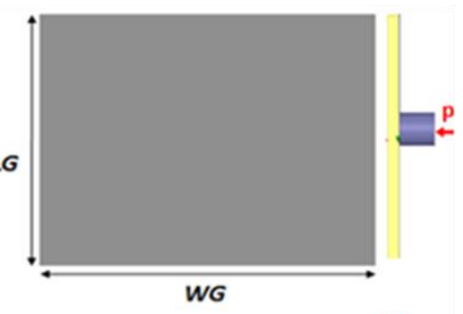

(b)

(c)

Figure 3. The proposed antenna: (a) In front view (b) bottom view 


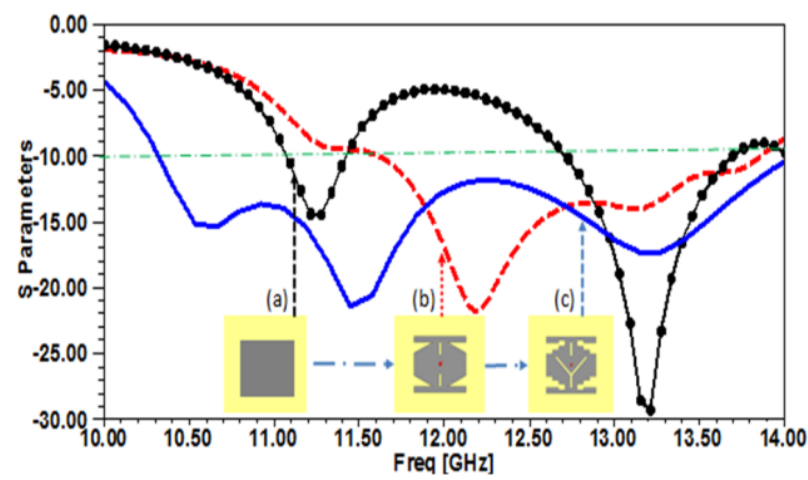

Figure 4. Reflection coefficient of the: (a) base antenna, (b) the rectangular slot antenna and (c) the final antenna

Table 1. Different values of antenna parameters

\begin{tabular}{cccc}
\hline Parameters & Values $(\mathrm{mm})$ & Parameters & Values $(\mathrm{mm})$ \\
\hline $\mathrm{W}_{\mathrm{S}}$ & 21.6 & $\mathrm{hs}$ & 1.6 \\
$\mathrm{~W}_{\mathrm{P}}$ & 12.2 & $\mathrm{i}$ & 1.2 \\
$\mathrm{Ls}$ & 17.6 & $\mathrm{f}$ & 3 \\
$\mathrm{~L}_{\mathrm{p}}$ & 10 & $\mathrm{~T} 2$ & 2.5 \\
$T 1$ & 3 & $r$ & 4 \\
$b$ & 1 & $y$ & 5 \\
$c$ & 1.65 & $W g$ & 21.5 \\
$S$ & 1.9 & $U$ & 4.25 \\
\hline
\end{tabular}

The final antenna offers a signal below $-10 \mathrm{~dB}$ in a bandwidth which ranges from $10.2 \mathrm{GHz}$ to $14 \mathrm{GHz}$ with a relative band of more than $137 \%$. We therefore conclude that the changes led to a good UWB structure. In the following, we propose to discuss the performance of our antenna.

The Figure 5 shows the gain 3D diagrams of the final antenna for the extreme frequencies of our band, as well as the central frequency $11.7 \mathrm{GHz}$. We note that the radiation pattern of our antenna is directional and has a main lobe vertical to the ground plane with a gain greater than $5 \mathrm{dBi}$. The side lobes are quite small. It can also be seen that the radiation intensity is oriented towards the vertical direction of the radiating element.

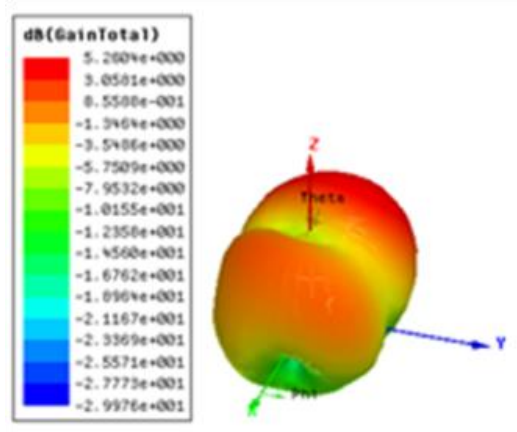

(a)

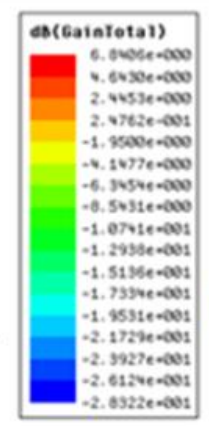

(b)

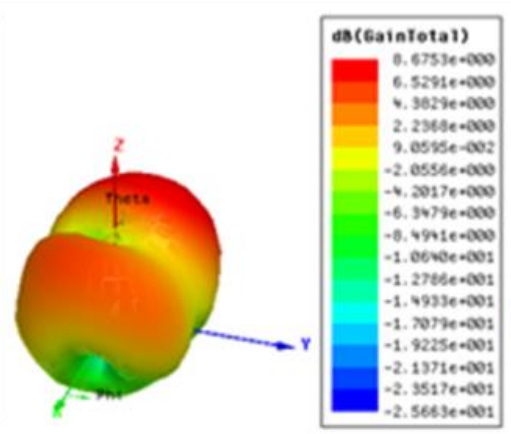

(b)

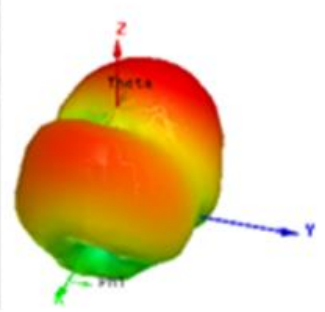

(c)

Figure 5. 3D radiation diagram of the proposed antenna at: (a) the frequency $10.7 \mathrm{GHz}$, (b) the frequency $11.7 \mathrm{GHz}$ and (c) the frequency $12.7 \mathrm{GHz}$

The Figure 6 illustrates the variation of the antenna gain as a function of the frequency. The antenna offers very good results with the characteristic of increasing with the frequency in the desired frequency band, with a maximum gain of approximately $8 \mathrm{dBi}$. The Figure 7 illustrates the radiation diagram of the antenna proposed in polar representation at different frequencies, namely $10.7 \mathrm{GHz}, 11.7 \mathrm{GHz}$ and $12.7 \mathrm{GHz}$ for the two planes $\mathrm{H}$ and $\mathrm{E}$. like most of the monopole antennas printed UWB, our antenna has radiation quasi-omnidirectional which is relatively stable in the whole band that interests us. It is noted from Figure 8 
the good efficiency of our final antenna, whose values are more than $86 \%$ in the entire operating band from $10.7 \mathrm{GHz}$ to $12.7 \mathrm{GHz}$. This indicates that the energy injected into the antenna is almost completely radiated.

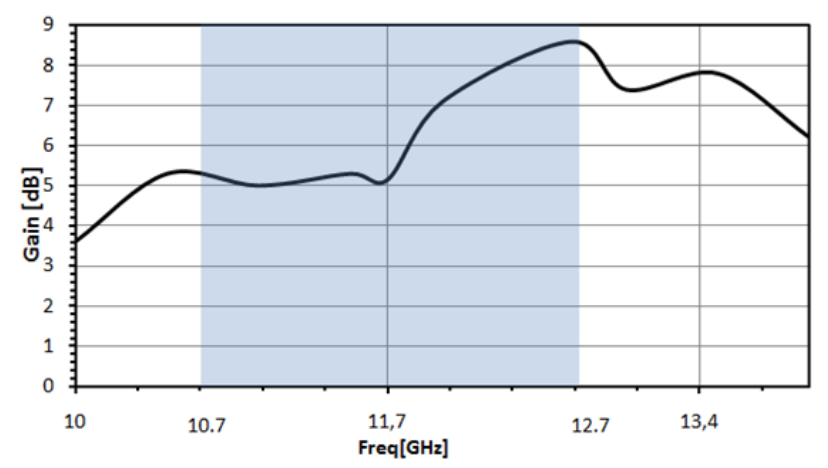

Figure 6. Gain of the proposed antenna versus frequency

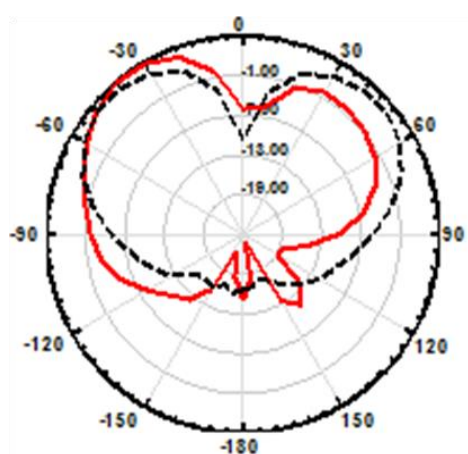

(a)

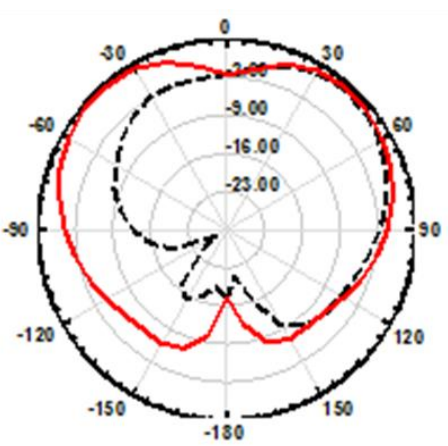

(b)

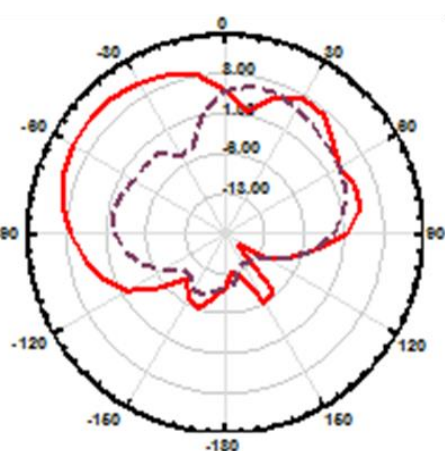

(c)

Figure 7. Antenna radiation pattern for different frequencies: (a) $\mathrm{F}=10.7 \mathrm{GHz}$, (b) $\mathrm{F}=11.7 \mathrm{GHz}$ and (c) $\overline{\mathrm{F}}=12.7 \mathrm{GHz}$

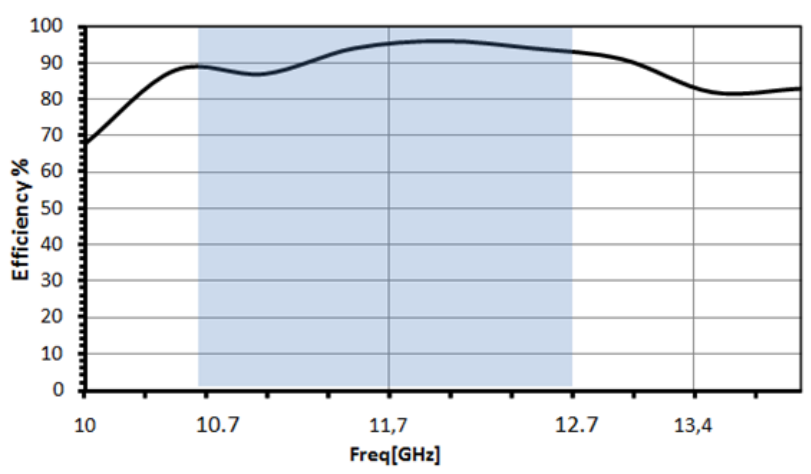

Figure 8. Proposed antenna efficiency

The plane $\mathrm{E}$ is defined as being the plane containing the vector of electric field as well as the maximum direction of radiation whereas the plane $\mathrm{H}$ containing the vector of magnetic field as well as the maximum direction of radiation [26, 27]. The Figure 9(a), gives us the distribution of $\mathrm{E}$ field with a maximum value obtained of $3.0910^{3} \mathrm{~V} / \mathrm{m}$ and Figure 9 (b) gives the distribution of $\mathrm{H}$ field with a maximum value obtained of $16.77 \mathrm{~A} / \mathrm{m}$. 


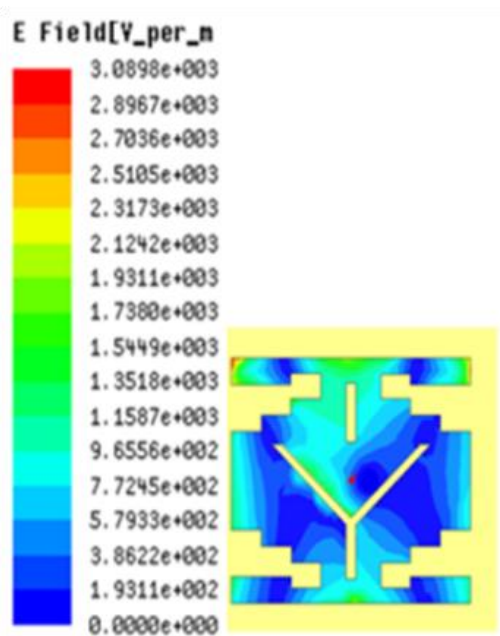

(a)

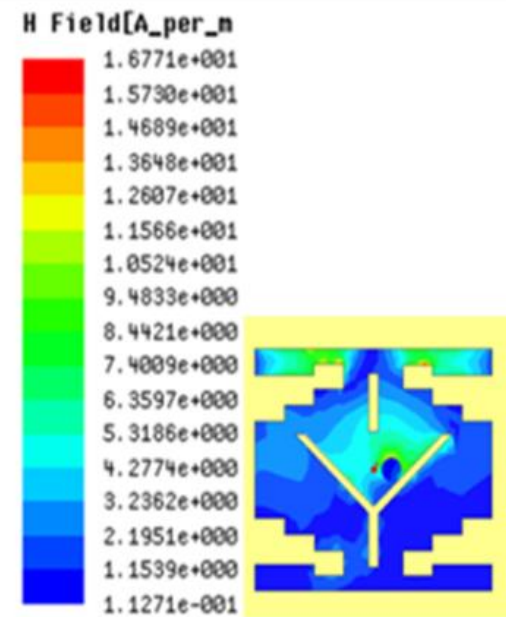

(b)

Figure 9. Distribution: (a) E field, (b) $\mathrm{H}$ field at the frequency $11.7 \mathrm{GHz}$ of the proposed antenna

\section{METAMATERIAL DECOUPLING STRUCTURE DESIGN}

We are going to study the square split ring resonator (SRR) represented by the Figure 10. The SRR will be sized for operation in the band ranging from $10.7 \mathrm{GHz}$ to $12.7 \mathrm{GHz}$. This resonator is made of copper deposited on a dielectric substrate of the FR4-epoxy type characterized by a permittivity of 4.4 and tangential losses of 0.02 with a thickness of $1.6 \mathrm{~mm}$. The studied square SRR is external side equal to $2.6 \mathrm{~mm}$, the two rings are concentric and spaced $0.15 \mathrm{~mm}$, the track width is $0.2 \mathrm{~mm}$, and a cut in one of these sides has a gap of $0.31 \mathrm{~mm}$. The dimensions of the structure are illustrated in Table 2 . The boundary conditions are applied for the simulations carried out. Magnetic walls along the $\mathrm{x}$ axis and Electric walls are applied along the $\mathrm{y}$ axis to get propagation along the $\mathrm{z}$ axis.

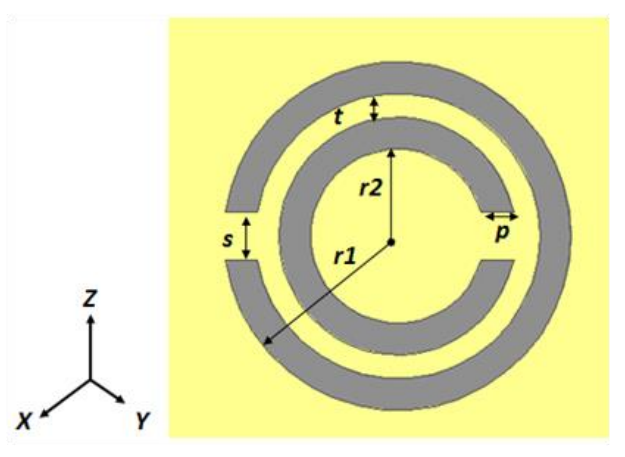

Figure 10. Geometrical parameters of metamaterial unit cell

Table 2. Different parameters and values of the antenna

\begin{tabular}{cccc}
\hline Parameters & Values $(\mathrm{mm})$ & Parameters & Values $(\mathrm{mm})$ \\
\hline $\mathrm{s}$ & 0.31 & $r_{1}$ & 1.3 \\
$\mathrm{p}$ & 0.2 & $r_{2}$ & 0.75 \\
$\mathrm{t}$ & 0.15 & & \\
\hline
\end{tabular}

The Figure 11 represents the reflection coefficients S11 and transmission S21 in $\mathrm{dB}$ following the simulation of the circular SRR presented in Figure 10. We notice that the SRR has a transmission of $-18.2 \mathrm{~dB}$ for a frequency of $11.7 \mathrm{GHz}$. This resonance is a magnetic and electrical resonance which is obtained following a penetration of the field through the rings and produces an induced current circulating on the rings and also excites the cuts of the rings. 
Based on the homogenization procedure, the real and imaginary parts of the effective permeability are calculated from the matrix $\mathrm{S}$ and are illustrated in Figure 12. We notice that the simulated structure presents a real part of the permeability real $\left(\mu_{e f f}\right)$ negative (from 11.2 to $12.15 \mathrm{GHz}$ ) around the resonance frequency $(11.75 \mathrm{GHz})$. As mentioned in Figure 13, the electric field plot is plotted in the SRR plan for the resonant frequency $11.7 \mathrm{GHz}$. We can notice that the field is maximal in the gap of the outer ring which is resonant. This figure highlights the capacitive effect between the rings, which explains the accumulation of the electric field in the opening of the ring.

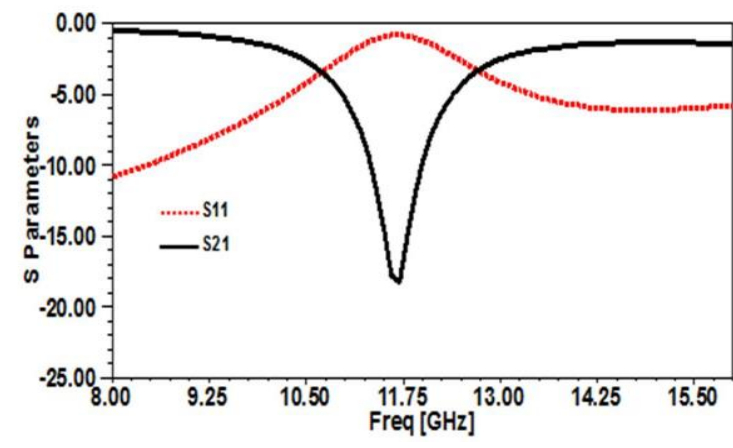

Figure 11. Circular SRR with E along the y axis: Transmission coefficient and reflection in $\mathrm{dB}$

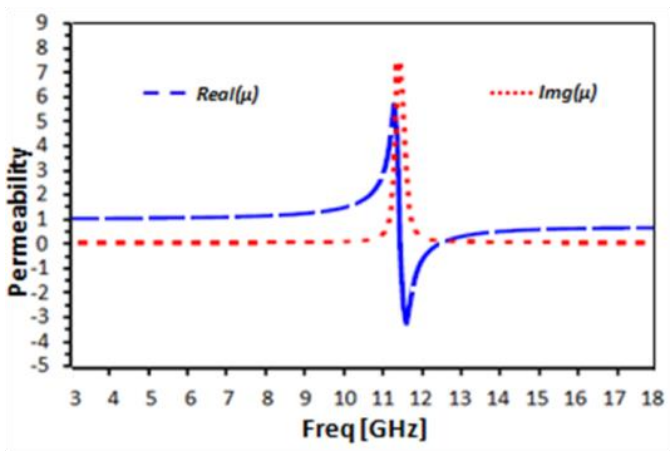

Figure 12. Real and imaginary part extracted from the relative permeability compared to the frequency

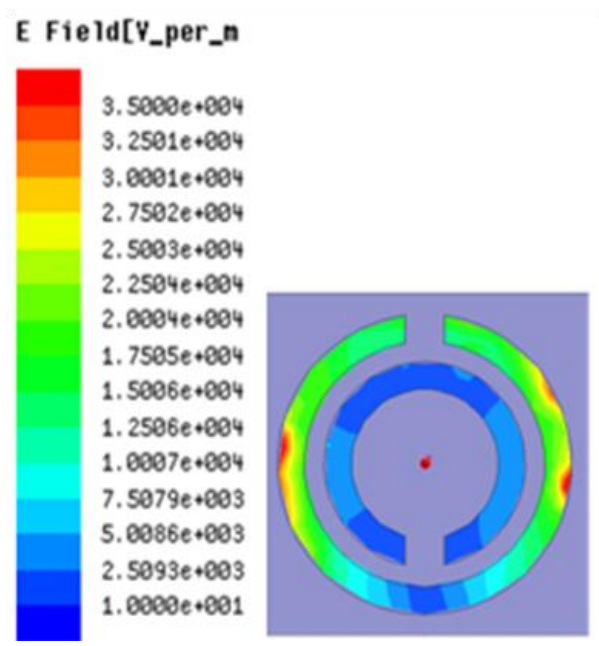

Figure 13. Capture of the electric field of a circular SRR at the frequency $11.7 \mathrm{GHz}$

In order to guarantee isolation across our entire frequency band, a parametric study be performed on the circular SRRs resonator allowed us to better understand the effect of all the parameters of the resonator and more precisely in this paper on the size of the opening of SRR in order to see the influence of this variation on the resonant frequency of SRR. From the simulation result mentioned in Figure 14, we notice that we have a proportional relationship between the increasing in the opening for the two rings of our SRR and the resonant frequency that changes with the opening. We can design resonators operating in welldefined frequencies.

In this part, we are going to study the influence of a circular SRR cell network between the two adjacent antennas in the first step in Figure 15. Therefore for this a network of SRR cells composed of 4 well-studied circular SRR cells is presented in Figure 16. We searched to present an electromagnetic blackberry for ensuring isolation on the fluxes and radiation interferences coming from the proximity of the adjacent antennas. Then a proposed solution based on the use of the metamaterial was presented which allows stopping or minimizing the propagation of surface waves, thus reducing mutual coupling in a MIMO antenna. 


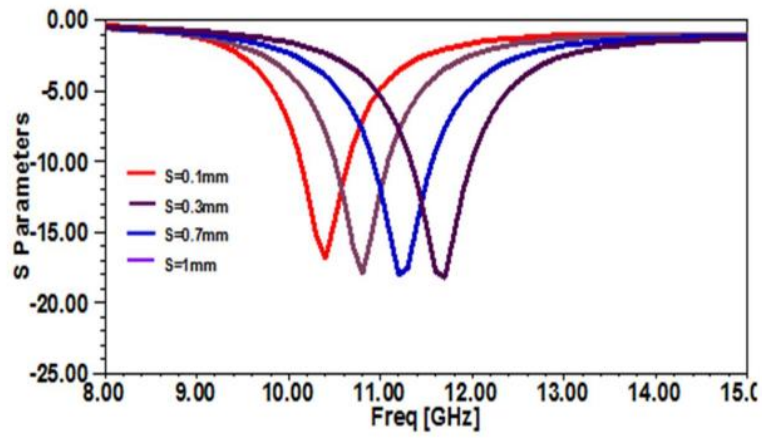

Figure 14. Reflection coefficient for different aperture values in the circular SRR
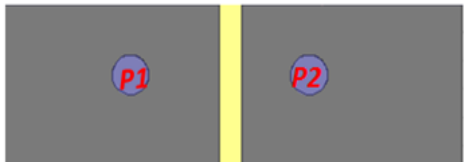

(a)<smiles>[Y]C([Y])[C]</smiles>

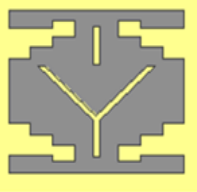

(b)

Figure 15. Structure of two patches without metamaterial: (a) front view and (b) bottom view

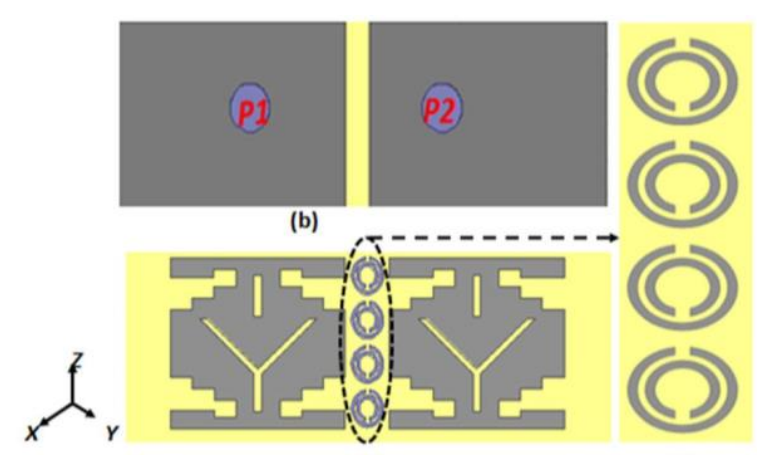

(a)

(c)

Figure 16. Structure of two patches : (a) in front view, (b) and bottom view, (c) Geometrical parameters of metamaterial 4 unit cells in front view

The structure of the two-element MIMO antenna was presented with two separate power ports, port 1 and port 2. Figure 17 presents the simulated of reflections coefficient S11 of our MIMO antenna proposed before and after the use of metamaterial. We noticed from Figure 16 that the reflections coefficient of the MIMO antenna with and without metamaterial is almost identical over the entire operating band which is initially presented and that each of them is below $-10 \mathrm{~dB}$.

The mutual coupling measured in terms of transmission coefficient at the frequency of $10.7 \mathrm{GHz}$ was initially $-14.4 \mathrm{~dB}$ and at the frequency $12.7 \mathrm{GHz}$ was $-19.7 \mathrm{~dB}$ between antenna 1 and antenna 2 . Between these antennas, the isolation values for the two limit frequencies of our operating band becomes more improved to get the value of $-29.4 \mathrm{~dB}$ and $-32.3 \mathrm{~dB}$ with the technique of coupling based on metamaterial which is used between the two antennas. Thus, the condition of having a mutual coupling is imposed less than $-20 \mathrm{~dB}$. The presented observation in Figure 18 of the transmission coefficient show a good quality of transmission with low power loss over the entire band in order to maintain the performance of a MIMO system. The isolation more notable than $28 \mathrm{~dB}$ over the entire band is obtained. This explains the isolation performance between the antennas with this planned structure that involves increasing the performance of the MIMO antennas.

In other words, the compound isolation of a chain of circular SRRs acts as an isolation to reduce the level of mutual coupling has been presented between the radiating elements. They ensure the rejection of all the unwanted frequency ranges which is further clarified by analyzing the distributions of surface currents with and without metamaterials at $11.7 \mathrm{GHz}$. Indeed, it is obvious that the use of the recommended solution considerably minimizes the level of surface currents for the two antennas at the operating frequency 11.7 GHz. Figure 19(a), mentions that the excited antenna (antenna $\mathrm{N}^{\circ} 1$ ) creates surface currents that go towards the non-excited antenna (antenna $\mathrm{N}^{\circ} 2$ ). So despite the fact that only one antenna is excited, we notice that a current also exists on the other non-excited element. The level of the maximum currents on the non-excited antenna is not equal to the one of the excited antenna but it is significant enough to increase the coupling between elements. After the use of the circular SRRs between the two elements as shown in the Figure 19(b), we observe that there is not the same current flow on the element $\mathrm{N}^{\circ} 2$. This implies that there is not an 
excessive distribution of current from the one worm to the other and consequently little of transfer of current between the two elements that leads to good mutual isolation.

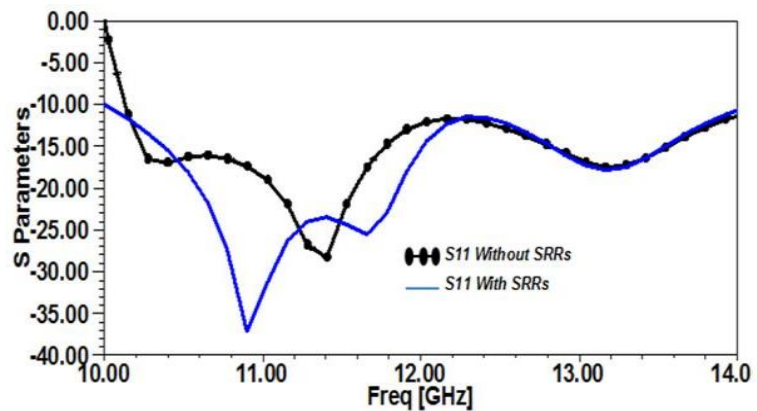

Figure 17. Return loss of the array structure without and with metamaterial

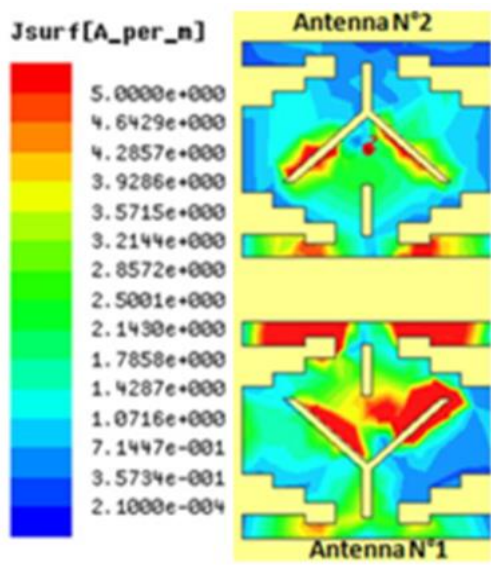

(a)

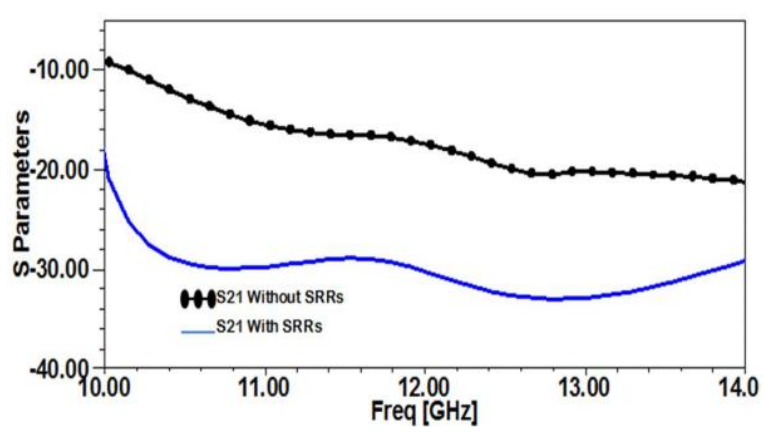

Figure 18. Isolation analysis of MIMO antennas without and with metamaterial

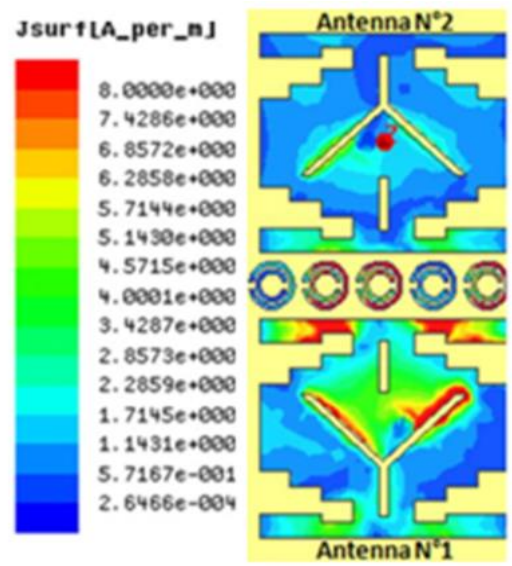

(b)

Figure 19. Distribution of surface currents of the two-element MIMO system at the frequency $11.7 \mathrm{GHz}$ : (a) without SRRs and (b) with SRRs

To validate the capacity and performance of the proposed UWB-MIMO antenna, it is necessary to have a low envelope correlation coefficient (ECC). The correlation envelope curve is presented in Figure 20, is calculated from the parameters $\mathrm{S}$ by the following relation [28].

$$
\mathrm{ECC}_{\mathrm{i}, \mathrm{j}}=\left|\frac{-\sum_{n=1}^{N} S_{n i}^{*} S_{n j}}{\sqrt{\left(1-\sum_{n=1}^{N}\left|S_{n i}\right|^{2}\right)\left(1-\sum_{n=1}^{N}\left|S_{n j}\right|^{2}\right)}}\right|^{2}
$$

Where $\mathrm{N}$ is number of ports, $S_{n i}$ and $S_{n j}$ are the $S$-parameters between ports $i$ and $j$ and the other ports of the system. In our case $\mathrm{N}=4$, and $\mathrm{i}=1$ (because the symmetry of our design). It indicates that the level obtained from the ECC correlation envelope not only always remains below 0.5 , but is also very low and does not exceed 0.04 in the worst case in the band [10.7-12.7] GHz. This satisfactory result shows that the signals are well decorrelated. It should also be noted that the diversity gain (DG) is a very important parameter and is very significant for the performance of the MIMO antenna. This parameter can be calculated using the following relation [29].

$$
D G=10 \sqrt{\left.1-(E C C)^{2}\right)}
$$




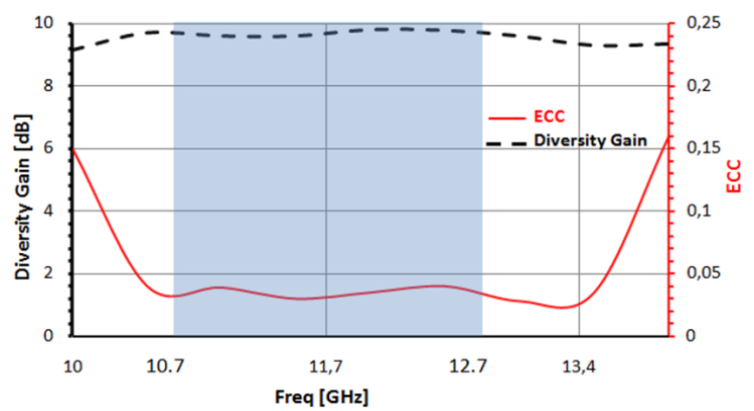

Figure 20. ECC and diversity gain of the proposed antenna

As the gain in diversity depends largely on the correlation envelope, then here again we notice in Figure 20 that the gain is at its maximum value (around $10 \mathrm{~dB}$ ) exactly at the points where the ECC is therefore better where it is as low as possible throughout the strip. In the second part we will study the influence of a circular SRR cell network applied between four antennas constituting our MIMO system, placed on the highest point of the substrate as shown in Figure 21.

The idea is to integrate two groups of SRRs composed of two chains above the substrate. The first chain includes 4 circular SRRs units on the horizontal axis for the separation of the opposing antennas two by two. The other chain includes 5 SRRs units placed on the vertical axis separating the adjacent antennas. These SRRs have been used to improve the isolation of adjacent, opposite and symmetrical antennas. Therefore an electromagnetic blackberry which arises and which minimizes or stops the propagation of surface waves, thus reducing mutual coupling in MIMO multi-antennas. This configuration is illustrated in Figure 21. The structure of the MIMO antenna with four elements has been presented with four supply ports separately, port 1 , port 2 , port 3 and port 4 Figure 21 . The Figures 22 present the simulated reflections coefficients (Sii) of our antenna MIMO offered before and after the use of metamaterial. Noting well from Figure 22 that the coefficients of the reflections of the MIMO antenna with and without metamaterial is almost identical over the entire operating band initially presented with a slight modification and that each of them is below $-10 \mathrm{~dB}$.
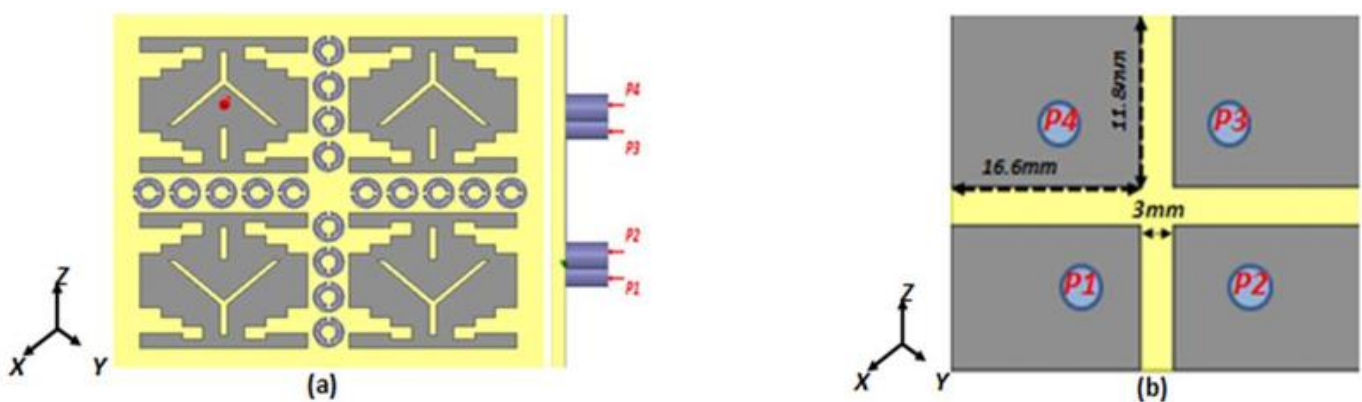

Figure 21. Structure of four patches antennas: (a) in front view, (b) and bottom view

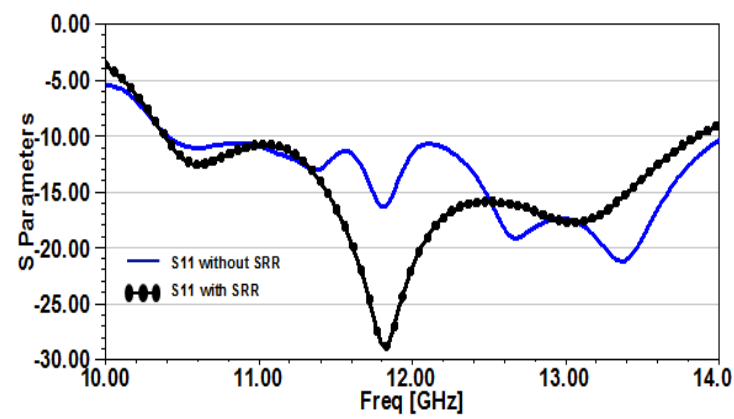

Figure 22. Return loss of the array structure without and with SRRs 
In order to obtain an efficient multi-element antenna system with high insulation between the power ports, we have suggested the use of a left hand MNG type metamaterial. The results of simulation of the transmission coefficients of the MIMO system with and without circular SRRs are mentioned in the Figures 23, 24 and 25. In addition, it is obvious that the proposed solution can guarantee better isolation between the four radiating elements. Indeed, the simulated mutual coupling with SRRs is equal to $-39 \mathrm{~dB}$ and $-28.5 \mathrm{~dB}$ in comparison with $-15.6 \mathrm{~dB}$ and $-22 \mathrm{~dB}$ initially at $10.7 \mathrm{GHz}$ and $12.7 \mathrm{GHz}$ respectively for the two adjacent elements (port 1-port 2). We also notice the same thing between the two opposite elements (port 1port 3) with a mutual coupling varies between $-38.3 \mathrm{~dB}$ and $-64.4 \mathrm{~dB}$ with the application of SRRs. A more significant gain in isolation protection over the entire strip is obtained with this planned structure.

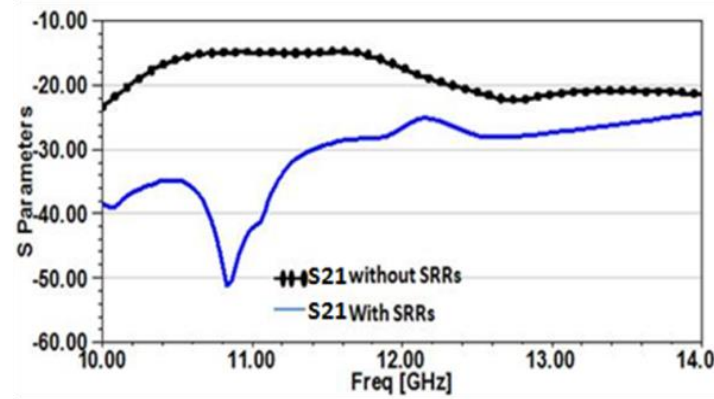

Figure 23. Isolation analysis of MIMO antennas (port 1-port 2) with and without metamaterial

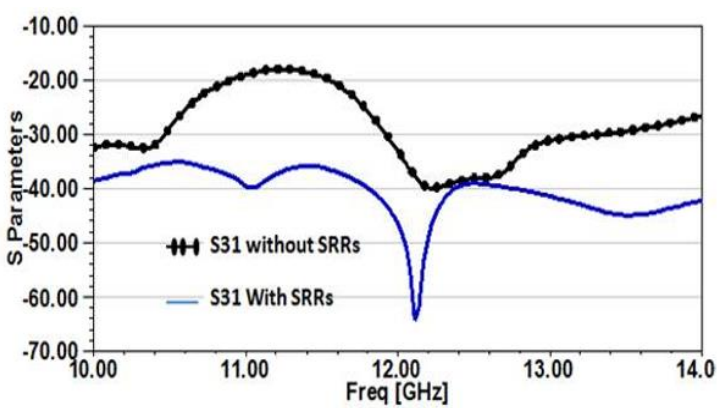

Figure 24. Isolation analysis of MIMO antennas (port 1-port 3) with and without metamaterial

The Figure 25 shows that the excited antenna creates surface currents which go towards those not excited. Indeed, as indicated in Figure 25 . Despite the fact that only one antenna is excited (here $n^{\circ} 1$ ), we can notice a current (in green or in red) on the three other non-excited elements. Thus the hot spots (in red) also exist on at least one of the other non-excited radiating elements (2, 3 or 4$)$. The level of the maximum currents on the non-excited antennas is not equal to that of the excited antenna, but nevertheless it is significant enough to increase the coupling between elements. This generates a strong coupling translated by values of parameters S21, S31 and S41 and therefore the value of the isolation obtained is not sufficient for a powerful multi-antenna system. This is why we will try to improve it by an implementation of a new configuration while keeping the antennas in their initial positions.

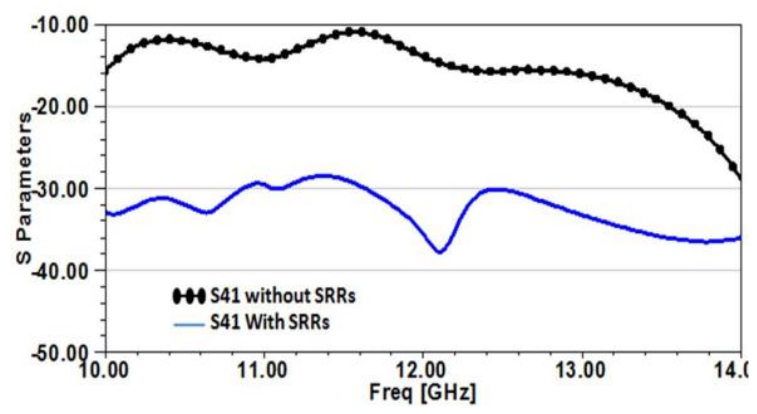

Figure 25. Isolation analysis of MIMO antennas (port 1-port 4) with and without metamaterial

The visualization of the surface currents on the radiating elements and on the ground plane with the use of the SRRs in Figure 26 clearly shows that when, by exciting port 1, for example, the maximum current is on it. It is then absorbed by the SRRs which prevents this current from arriving on the other non-excited ports and therefore makes it possible to reduce the coupling between accesses.

In Figure 26(a), we see that the total simulated efficiency of a single antenna put in MIMO system without application of SRRs is between $62 \%$ and $73 \%$ over the whole operating band. The maximum total efficiencies of the different antennas of the reference system are low due to the low isolation between these antennas. Our objective is therefore to use the metamaterial technique to increase the isolation between the 
antennas. Given the fact that the antennas are symmetrical, only the efficiency of a single antenna is presented each time. So increasing the isolation between the antennas improves their overall efficiencies. According to the Figures 23, 24 and 25 the minimum value of the isolation is $-23 \mathrm{~dB}$ which seems interesting to have a good total efficiency. We can see clearly on curve Figure 26 (b), that the total efficiencies of MIMO system with insertion of SRRs are higher than those of the initial system without SRRs and that in the entire desired operating band. In the best of cases, the gain of efficiency in \% provided, by the isolation technique applied with the use of SRRs, on the total efficiency is $+18 \%$ see Figure 27.

The Figure 28 shows the results obtained in simulation of the ECC correlation envelope and the diversity gain of port $n^{\circ} 1$ compared to accesses 2,3 and 4 . The system being symmetrical, the results shown below of port $n^{\circ} 1$ are exactly the same for the other three accesses. At the level of the operating band the ECC correlation envelope is very low and it is around 0.1 in the worst case and it does not exceed the value of 0.5 . As for the gain in diversity, it is very high in terms of operating frequencies and hovers around $10 \mathrm{~dB}$.

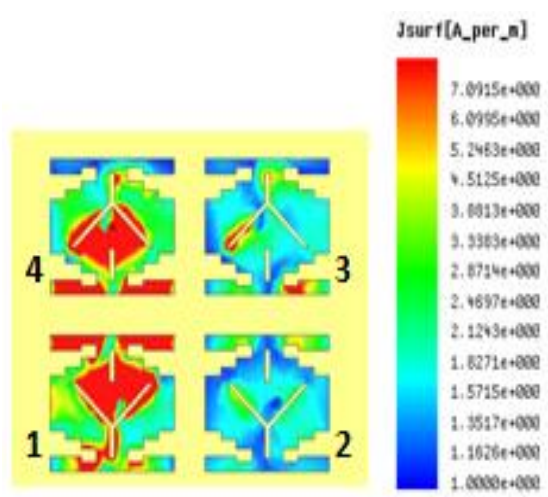

(a)

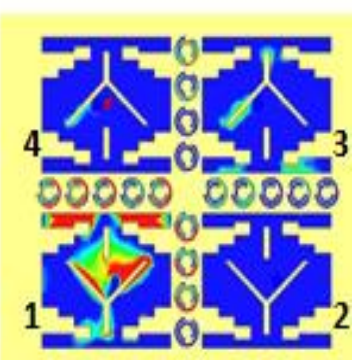

(b)

Figure 26. Surface currents for antenna 1 at $11.7 \mathrm{GHz}$ : (a) without, (b) with SRRs

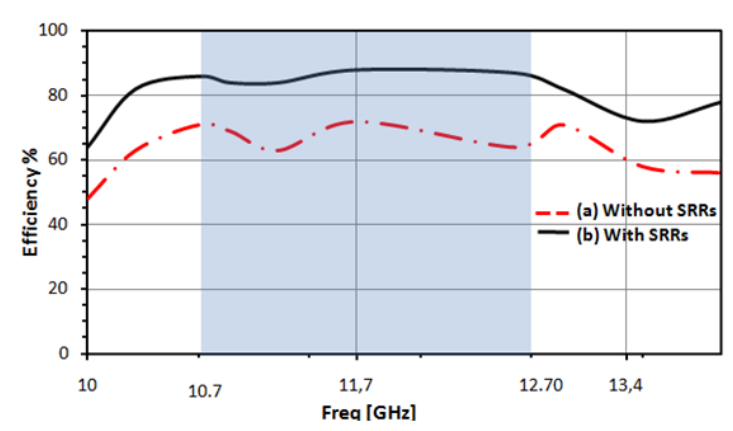

Figure 27. Total simulated efficiency without and with SRRs

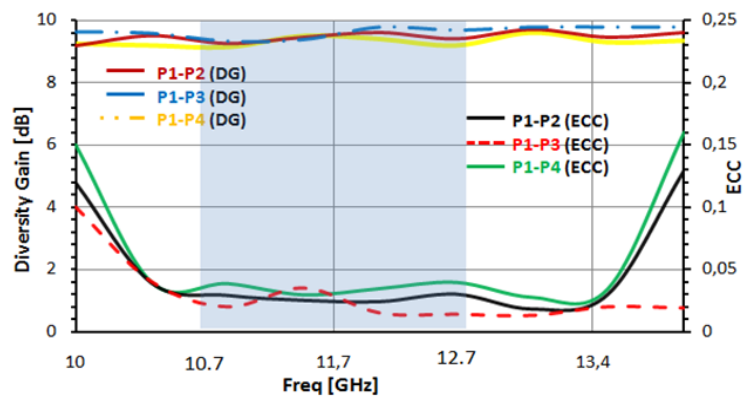

Figure 28. ECC and DG simulated from parameters $\mathrm{S}$ with SRRs, for access 1 compared to the other three accesses 2, 3 and 4 successively

\section{CONCLUSION}

In this article, the studies and research that have been discussed have focused on the design, optimization and performance evaluation of two and four element MIMO systems based on metamaterials for communication networks applied to antennas UWB. The simulation results of the final system developed confirmed the advantages of the proposed idea not only from the point of view of mutual coupling but also in terms of weight reduction, size and gain in diversity. The suggested antenna presents a good diversity performance with ECC $<0.04$ and diversity gain $(\mathrm{DG}>9 \mathrm{~dB})$ and the efficiency is around $86 \%$. This is beneficial for their integration into telecommunications systems.

\section{ACKNOWLEDGEMENTS}

This paper is supported by the R\&D pole of the EITA Consulting Company. 


\section{REFERENCES}

[1] Federal Communications Commission, "First Order and Report, Revision of Part 15 of the Commission's Rules Regarding UWB Transmission Systems," FCC 02-48, 2002.

[2] B. H. G. Gao, L. He, S. Wang, and C. Yang, "Investigation of A Reconfigurable Dual Notched UWB Antenna by Conceptual Circuit Model and Time-Domain Characteristics," Microwave and Optical Technology Letters, vol. 59, no. 6, pp. 1326-1332, 2017.

[3] R. B. Rani and S. K. Pandey," A CPW-Fed Circular Patch Antenna Inspired by Reduced Ground Plane and CSRR Slot for UWB Applications with Notch Band," Microwave and Optical Technology Letters, vol. 59, no. 4, pp. 745-749, 2017.

[4] M. S. Sharawi, S. K. Podilchak, M. T. Hussain and Y. M.M. Antar, "Dielectric Resonator Based MIMO Antenna System Enabling Millimeter-Wave Mobile Devices," IET Microwaves, Antennas and Propagation, vol. 11, no. 2, pp. 287-293, Jan. 2017.

[5] W. Xiao, T. Mei, Y. Lan, Y. Wu, R. Xu and Y. Xu, "Triple band-notched UWB monopole antenna on ultra-thin liquid crystal polymer based on ESCSRR," Electronics Letters, vol. 53, no. 2, pp. 57-58, 2017.

[6] S. Cumali and N. Tayfun, "Design and characterization of a resonator-based metamaterial and its sensor application using microstrip technology," Optical Engineering, vol. 55, no. 2, 2016, doi: 10.1117/1.OE.55.2.027107.

[7] C. Abdelhamid, H. Sakli, "Mutual Reduction in the Coupling of the MIMO Antenna Network Applied to the Broadband Transmission," Advances in Science, Technology and Engineering Systems Journal, vol. 5, no. 2, pp. 338-343, 2020.

[8] R. N. Tiwaria, P. Singh, and B. K. Kanaujia, "A modified microstrip line fed compact UWB antenna for WiMAX/ISM/WLAN and wireless communications," AEU-International Journal of Electronics and Communications, vol. 104, pp. 58-65, May 2019.

[9] A. Iqbal, O. A. Saraereh, A. W. Ahmad, S. Bashir, "Mutual Coupling Reduction Using F-Shaped Stubs in UWBMIMO Antenna," IEEE Access, vol. 6, pp. 2755-2759, 2018.

[10] H. Yu, G. Yang, F. Meng, and Y. Li, "Performance analysis of MIMO system with single RF link based on switched parasitic antenna," Symmetry, vol. 9, no. 12, 2017, Art. no. 304.

[11] K. Wei, J. Li, L. Wang, Z. Xing, and R. Xu, "Sshaped periodic defected ground structures to reduce microstrip antenna array mutual coupling," Electronics Letters, vol. 52, no. 15, pp. 1288-1290, 2016.

[12] K. Wei, J. Li, L. Wang, Z. Xing, and R. Xu, "Mutual coupling reduction by novel fractal defected ground structure bandgap filter," IEEE Transactions on Antennas and Propagation, vol. 64, no. 10, pp. 4328-4335, 2016.

[13] T. Jiang, T. Jiao, and Y. Li, "Array mutual coupling reduction using L-loading e-shaped electromagnetic band gap structures," International Journal of Antennas and Propagation, vol. 2016, 2016.

[14] T. Jiao, T. Jiang, and Y. Li, "A low mutual coupling reduction of a MIMO antenna array using 3-D electromagnetic isolation wall structures," 6th Asia-Pacific Conference on Antennas and Propagation, Xi'an, China, 2017.

[15] T. Jiang, T. Jiao, Y. Li, and W. Yu, "A low mutual coupling MIMO antenna using periodic multilayered electromagnetic band gap structures," Applied Computational Electromagnetics Society Journal, vol. 33, no. 3, pp. 305-311, 2018.

[16] S. Zhang and G. Pedersen, "Mutual coupling reduction for UWB MIMO antennas with a wideband neutralization line," IEEE Antennas and Wireless Propagation Letters, vol. 15, pp. 166-169, 2016.

[17] A. Ramachandran, S. Mathew, V. Rajan, and V. Kesavath, "A compact triband quad-element MIMO antenna using SRR Ring for High Isolation," IEEE Antennas Wirel. Propag. Lett, vol. 16, pp. 1409-1412, 2017.

[18] M. S. Sharawi, M. Ikram, and A. Shamim, "A Two Concentric Slot Loop Based Connected Array MIMO Antenna System for 4 G/5 G Terminals," IEEE Trans. Antennas Propag. vol. 62, no. 12, pp. 6679-6686, Feb. 2017.

[19] A. Iqbal, O. A Saraereh, A. Bouazizi, and A. Basir, "Metamaterial based highly isolated mimo antenna for portable wireless applications," Electronics, vol. 7, no. 10, 2018, Art. no. 267.

[20] M. S. Sharawi, S. K. Podilchak, M. U. Khan, and Y. M. Antar, "Dual-frequency DRA-based MIMO antenna system for wireless access points," IET Microwaves, Antennas \& Propagation, vol. 11, no. 8, pp. 1174-1182, 2017.

[21] A. Iqbal, A. Basir, A. Smida, N. K. Mallat, I. Elfergani, J. Rodriguez et al., "Electromagnetic bandgap backed millimeter-wave mimo antenna for wearable applications," IEEE Access, vol. 7, pp. 111135-111144, 2019.

[22] Y. Fu and G.-M. Yang, "Design of compact multiband MIMO antenna for the mobile handsets," Microw. Opt. Technol. Lett., vol. 58, no. 10, pp. 2411-2415, Oct. 2016.

[23] W. Hong, "Solving the 5G mobile antenna puzzle: assessing future directions for the $5 \mathrm{G}$ mobile antenna paradigm shift," IEEE Microwave Magazine, vol. 18, no. 7, pp. 86-102, 2017.

[24] K.-L. Wong, Y.-C. Chen, and W.-Y. Li, "Four LTE low-band smartphone antennas and their MIMO performance with user's hand presence," Microwave and Optical Technology Letters, vol. 58, no. 9, pp. 2046-2052, 2016.

[25] J. Choi, W. Hwang, C. You, B. Jung, and W. Hong, "Four-element reconfigurable coupled loop MIMO antenna featuring LTE full band operation for metallic-rimmed smartphone," IEEE Transactions on Antennas and Propagation, vol. 67, no. 1, pp. 99-107, 2019.

[26] J.-Y. Lee, S.-H. Kim, and J.-H. Jang, "Reduction of mutual coupling in planar multiple antenna by using 1-D EBG and SRR structures," IEEE Transactions on Antennas and Propagation, vol. 63, no. 9, pp. 4194-4198, 2015.

[27] Chandel, R., A. K. Gautam, and K. Rambabu, "Tapered fed compact UWB MIMOdiversity antenna with dual band notched characteristics," IEEE Transactions on Antennas and Propagation, vol. 66, pp. 1677-1684, 2018.

[28] Huang, C., C. Ji, X.Wu, J. Song, and X. Luo, "Combining FSS and EBG surfaces for high-efficiency transmission and low-scattering properties," IEEE Transactions on Antennas and Propagation, vol. 66, pp. 1628-1632, 2018. 
[29] J.-Y. Lee, S. Jung, Y. Youn, J. Park, W. Kwon, and W. Hong, "Optically transparent 1-D EBG antenna using subskin depth thin-film alloy in the Ka-band," 2019 13th European Conference on Antennas and Propagation (EuCAP), Krakow, Poland, 31 March-5 April, 2019, pp. 1-3.

[30] J. Park, S. Y. Lee, Y. Kim, J. Lee, and W. Hong, "Hybrid antenna module concept for $28 \mathrm{GHz} 5 \mathrm{G}$ beamsteering cellular devices," 2018 IEEE MTT-S International Microwave Workshop Series on 5G Hardware and System Technologies (IMWS-5G), Dublin, Ireland, 30-31 Aug. 2018, pp. 1-3.

[31] A. M. Elshirkasi, A. A. Al-Hadi, M. F. Mansor, R. Khan and P. J. Soh, "Envelope Correlation Coefficient of a Two-Port MIMO Terminal Antenna under Uniform and Gaussian Angular Power Spectrum with User's Hand Effect," Progress In Electromagnetics Research C, vol. 92, pp. 123-136, 2019.

\section{BIOGRAPHIES OF AUTHORS}

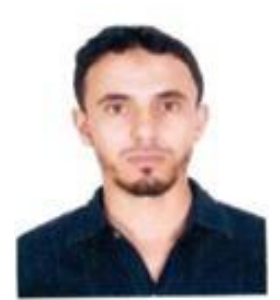

Chafai Abdelhamid is born in Tunisia in 1978. He is a research engineer in microwave engineering at the Center for Studies and Research of Telecommunications (CERT). His area of research is: RF and microwave. His area of interest is the design of hybrid active and passive monolithic microwave circuit, metamaterials, miniature antennas.

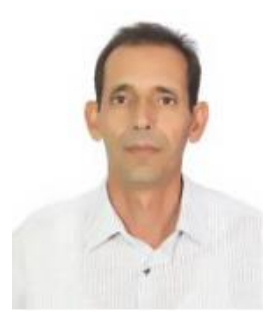

Hedi Sakli is born in Tunisia in 1966. He received the M.S. degree in High Frequency Communication Systems from Marne-La-Valley University, France in 2002, a PhD degree in 2009 and HDR degree in 2014 in telecommunications from the National Engineering School of Tunis, Tunis El Manar University, Tunisia. He is since 2010 assistant professor at the University of Gabes. In 2016 he is Associate Professor. He is the author of more than 50 papers. His research interests propagation in anisotropic media, Ferrite and metamaterials, numerical methods in electromagnetic, FSS, antennas, sensors, 5G, connected objects and sensor networks.

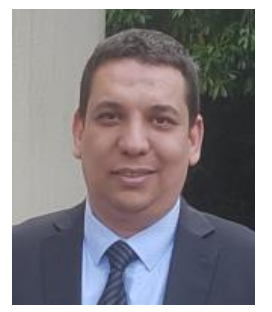

Nizar Sakli is born in Tunisia in 1981. President of EITA Consulting in Paris (France). His field of activity is computer science, $5 \mathrm{G}$, connected objects, IoT and sensor networks. 